\title{
Irregular Precoder-Aided Differential Linear Dispersion Codes
}

\author{
$\mathrm{Nan} \mathrm{Wu}$ \\ School of ECS, University of Southampton \\ SO17 1BJ, UK \\ nw04r@ecs.soton.ac.uk \\ http://www-mobile.ecs.soton.ac.uk/newcomms/
}

\author{
Lajos Hanzo \\ School of ECS, University of Southampton \\ SO17 1BJ, UK \\ lh@ecs.soton.ac.uk \\ http://www-mobile.ecs.soton.ac.uk/newcomms/
}

\begin{abstract}
In this treatise, we propose a novel family of serial concatenated IrRegular Convolutional Coded (IRCC) IrRegular Precoded Differential Linear Dispersion Codes (IR-PDLDC). The irregularity imposed on both the outer and inner codes allows the proposed scheme to operate across a wide range of SNRs, while maintaining an infinitesimally low BER. Each coding block of the IR-PDLDC scheme is designed with throughput maximization in mind, using Extrinsic Information Transfer (EXIT) charts. The proposed scheme is capable of providing a high throughput for SNRs in excess of $\rho=-12 \mathrm{~dB}$.
\end{abstract}

\section{INTRODUCTION}

Wireless communication systems using multiple antennas at both the transmitter and receiver, which are referred to as a Multiple Input and Multiple Output (MIMO) systems, have the potential of maintaining reliable transmissions at high data rates [1], [2]. The design of coding schemes for MIMO systems operating at high Signal-to-Noise Ratios (SNRs) involves a tradeoff between the achievable rate at which the system's capacity increases and the rate at which the error probability decays [3].

The set of coherently detected Linear Dispersion Codes (LDCs) [4], constitutes a wide-ranging class of space-time codes exhibiting diverse characteristics. Hence this family provides a natural framework for flexible system designs. The revolutionary concept of LDCs [4] [5] invokes a matrixbased linear modulation framework, where each space-time transmission matrix is generated by a linear combination of so-called 'dispersion' matrices used for dispersing or mapping the symbols to the transmit antennas, where the weights of the constituent matrices are determined by the transmitted symbols.

In practice, the knowledge of the Channel's Impulse Response (CIR) is typically acquired using a channel-sounding sequence. However, an excessive number of training symbols may be required, especially when numerous antennas are involved and/or when the Doppler frequency is high. Hence precious transmit power as well as valuable bandwidth is wasted. Furthermore, the coherently detected system typically suffers from a substantial performance penalty in the presence of realistic rather than idealistic, i.e. perfect channel estimation. Therefore, there is considerable interest in developing

The financial support of the EPSRC, UK, that of Vodafone in the framework of the Dorothy Hodgkin Postgraduate Award (DHPA) and of the EU is gratefully acknowledged. differentially encoded and non-coherently detected schemes dispensing with pilot-based channel estimation [6] [7] [8].

Serial Concatenated Codes (SCC) are capable of attaining an infinitesimally low BER, while maintaining a manageable decoding complexity [9]. Since LDCs have the ability to approach the potential capacity of MIMO systems [4], it is natural to serially concatenate for example a channel code as the outer code with the DLDCs employed as the 'inner code' in order to provide a high throughput without the burden of channel estimation.

It has been demonstrated in [10] [11] that SCCs benefit from having an open convergence tunnel at low SNRs, when IrRegular Convolutional Codes (IRCCs) are adopted as the outer channel code, since IRCCs exhibit flexible EXIT chart characteristics [10]. However, SCCs employing irregular outer codes are unable to operate at low SNRs, where the associated convergence tunnel is closed. Furthermore, nearcapacity IRCC schemes may require an excessive number of iterations at the receiver to achieve an infinitesimally low BER, which may exceed the affordable complexity budget of mobile handsets.

Motivated by the above-mentioned flexibility of the irregular outer code design philosophy, in this treatise we circumvent the IRCC-related outer code limitations by proposing IrRegular Precoded DLDCs (IR-PDLDC) as inner codes and serially concatenate the resultant IR-PDLDC with IRCCs in order to achieve an infinitesimally low BER, when employing iterative decoders. The rationale and novelty of the proposed iteratively detected irregular scheme are:

- We propose an IR-PDLDC as the inner code of SCCs, while using IRCCs as the outer code, which are capable of reliably operating even at low SNRs;

- We investigate the maximum throughput of the proposed scheme with the aid of EXIT charts [12], when using Minimum Mean Squared Error (MMSE) detectors;

- IR-PDLDCs may be reconfigured by activating different dispersion matrices at a modest hardware cost;

- The proposed scheme is suitable for arbitrary antenna configurations;

We commence our discourse by providing a detailed description of the proposed IRCC-coded IR-PDLDC system's structure in Section II. In Section III, we demonstrate how the proposed irregular system is constructed from a throughput 


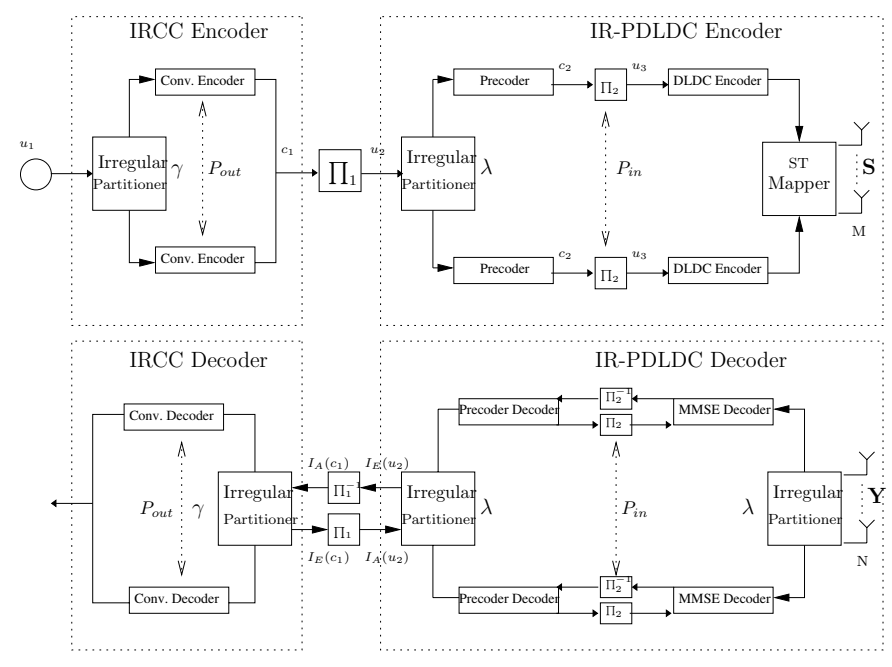

Figure 1. Schematic of the IRCC-coded IR-PDLDC using iterative decoding.

maximization perspective. Our simulation results are discussed in Section IV. Finally, we conclude our discourse in Section V.

\section{SYSTEM DESCRIPTION}

We consider a MIMO system employing $M$ transmit as well as $N$ receive antennas. Figure 1 portrays the system model of the proposed serially concatenated IRCC-coded IRPDLDC scheme. The irregularity is imposed on both the outer IRCC code and the inner IR-PDLDC code, where we have $P_{\text {out }}$ and $P_{\text {in }}$ number of outer and inner component codes, respectively. At the transmitter, a frame of information bits $u_{1}$ is encoded by an IRCC encoder. The fraction of the bitstream $u_{1}$, which will be fed feed into each of the IRCC's component codes is controlled by the "irregular partitioner" block of Figure 1 based on the weighting coefficient vector $\gamma=\left[\gamma_{1}, \cdots, \gamma_{P_{\text {out }}}\right]$. Then the encoded bits $c_{1}$ are interleaved by a random bit interleaver, yielding the outer encoded bits $u_{2}$. Again, the IR-PDLDC's "irregular partitioner" feeds the appropriately selected fraction of $u_{2}$ into the various PDLDC component codes, governed by the weighting coefficient vector $\boldsymbol{\lambda}=\left[\lambda_{1}, \cdots, \lambda_{P_{i n}}\right]$. Memory-1 unity-rate precoders were employed for all the PDLDC components. More explicitly, within each PDLDC encoder, the resultant precoded bits $c_{2}$ are interleaved by a second interleaver, yielding the interleaved bits $u_{3}$, which are then fed into the DLDC block of Figure 1. After Pulse-Amplitude Modulation (PAM), the $n$-th symbol vector $\mathbf{K}_{n}=\left[s_{1}, \cdots, s_{Q}\right]$ containing $Q$ PAM symbols is uniquely mapped to a space-time codeword $\mathbf{X}_{n} \in \zeta^{M \times T}$. Hence, the transmission matrix $\mathbf{S}_{n}$ generated by differential encoding is given by:

$$
\left\{\begin{array}{ccc}
\mathbf{S}_{n}= & \mathbf{I}_{n} & (n=1), \\
\mathbf{S}_{n}= & \mathbf{S}_{n-1} \cdot \mathbf{X}_{n} & (n>1),
\end{array}\right.
$$

where the identity matrix $\mathbf{I}_{n} \in \zeta^{M \times T}$ is transmitted as a reference, which contains no information. The differential encoding operation of Equation (1) requires $\mathbf{X}_{n}$ to be a unitary matrix, which results in $T=M$. Then, the "ST mapper' of Figure 1 maps $\mathbf{S}_{n}$ to $M$ spatial- and $T$ temporaldimensions. The DLDC obeying this configuration will be denoted as $\operatorname{DLDC}(M N T Q)$ and its rate becomes $R_{D L D C}=\frac{Q}{T}$ (sym/slot).

At the receiver, the $n$-th received signal matrix $\mathbf{Y}_{n}$ is related to $\mathbf{S}_{n}$ by:

$$
\mathbf{Y}_{n}=\sqrt{\frac{\rho}{M}} \mathbf{H}_{n} \mathbf{S}_{n}+\mathbf{V}_{n},
$$

where the CIR matrix $\mathbf{H}_{n}$ models an independent and identically distributed (i.i.d.) flat Rayleigh fading channel, which has a constant envelope and phase over $T$ channel use intervals and faded at the beginning of the next block. The correlation within each MIMO link is governed by the normalized Doppler frequency $f_{d}$. The noise matrix $\mathbf{V}_{n}$ is assumed to impose independent samples of a zero-mean unit-variance complexvalued Gaussian random process and $\rho$ denotes the SNR.

If we assume the channel varies slowly, then using Equations (1) and (2) we have:

$$
\begin{aligned}
\mathbf{Y}_{n} & =\sqrt{\frac{\rho}{M}} \mathbf{H}_{n} \mathbf{S}_{n-1} \mathbf{X}_{n}+\mathbf{V}_{n} \\
& =\mathbf{Y}_{n-1} \mathbf{X}_{n}+\mathbf{V}_{n}-\mathbf{V}_{n-1} \mathbf{X}_{n} .
\end{aligned}
$$

Note that $\mathbf{H}_{n}$ does not appear in the above equation. This implies that as long as the channel faded slowly, differentially encoded transmission facilitates detection without knowing the CIR. Hence, ML or MMSE detectors can be employed.

Again, the "irregular partitioner" of Figure 1 determines the specific portion of the received signal as well as the $a$ priori information to be detected by each component decoder of the IR-PDLDC and IRCC scheme according to $\lambda$ and $\gamma$, respectively. Then, an iterative decoding structure is employed, where extrinsic information is exchanged between the three Soft-In Soft-Out modules, namely the MMSE detector, the precoder and the outer IRCC decoder in a number of consecutive iterations. To be specific, in Figure 1, $I_{A}()$ denotes the a-priori information represented in terms of Log-Likelihood Ratios (LLRs), while $I_{E}()$ denotes the extrinsic information also expressed in terms of LLRs. Note that the intermediate rate-1 precoder processes two a-priori inputs, namely those arriving from the MMSE detector as well as those from the outer decoder and generates two extrinsic outputs. More detailed discussions on the iterative decoding process are provided in [13] [14].

\section{EXIT CHART BASED DESIGN OF IR-PDLDCS}

In our forthcoming EXIT chart analysis, the precoder's decoder and the MMSE decoder are considered as a single 'inner' decoding block, constituted by the IR-PDLDC's decoder seen in Figure 1. The advantage of this representation is that the IR-PDLDC block's extrinsic information output $I_{E}\left(u_{2}\right)$ is only determined by the received signal matrix $\mathbf{Y}_{n}$ and the a-priori input $I_{A}\left(u_{2}\right)$, but remains unaffected by the extrinsic information exchange between the precoder's decoder and the MMSE detector. Thus, we can project the three-stage system into a two-stage system and hence the traditional twodimensional EXIT charts [15] [16] are applicable. 
Following the approach of [16], the corresponding EXIT transfer function of the proposed inner IR-PDLDC system becomes:

$$
I_{E}\left(u_{2}\right)=\Gamma_{i n}\left[I_{A}\left(u_{2}\right), \rho\right] .
$$

In [10], the authors have shown that the aggregate EXIT function of an irregular code can be obtained from the linear combination of that of its component codes, under the assumption that the probability density function of the LLRs is symmetric and continuous. More explicitly, the EXIT function of the proposed inner IR-PDLDC scheme is given by:

$$
\Gamma_{i n}=\sum_{i=1}^{P_{i n}} \lambda_{i} \Gamma_{i}\left(I_{i n}, \rho\right),
$$

where $\Gamma_{i}$ denotes the EXIT function of the $i$-th PDLDC component.

\section{A. Generating unitary space-time matrices}

It is desirable that the unitary space-time matrix $\mathbf{X}_{n}$ of Equation (1) possesses the flexible linear structure of LDCs [5]. However, even if each dispersion matrix is unitary, there is no guarantee that their weighted sum becomes a unitary matrix. In [8], the authors proposed to design dispersion matrices in the Hermitian space and uniquely project the resultant matrix to another unitary matrix using the Cayley transform [8], since the Hermitian space is linear.

More explicitly, given the symbol vector $\mathbf{K}$, the $(M \times M)$ element space-time coded matrix is given by:

$$
\tilde{\mathbf{X}}_{n}=\sum_{q=1}^{Q} \mathbf{A}_{q} s_{q}, \quad q=1, \cdots, Q,
$$

where $\mathbf{A}_{q}$ denotes the $q$-th Hermitian dispersion matrix. Realvalued modulation schemes have to be employed, in order to ensure that $\tilde{\mathbf{X}}_{n}$ is also a Hermitian matrix. Hence, by employing the Cayley transform [8], the resultant unitary matrix becomes:

$$
\mathbf{X}_{n}=\left(\mathbf{I}-i \tilde{\mathbf{X}}_{n}\right)\left(\mathbf{I}+i \tilde{\mathbf{X}}_{n}\right)^{-1}
$$

where $\mathbf{X}_{n}$ is used during the differential encoding process of Equation (1). It has been shown that the Cayley transform [8] preserves the diversity gain and coding gain designed for $\tilde{\mathbf{X}}_{n}$. Observe in Equation (3) that the associated linear relationship ensures that the DLDCs inherit the LDCs' properties. Most importantly, the DLDC's maximum achievable diversity order is given by $N \cdot \min (M, T)$.

\section{B. Generating DLDC component codes}

In this section, we demonstrate how to generate an inner IR-PDLDC coding scheme containing $P_{i n}=6$ components for a MIMO configuration having $M=3$ transmit and $N=2$ receive antennas. Since we have $T=M$, the resultant DLDC has the potential of achieving the maximum diversity order of $D=6$. By setting $Q=1$, we have $\operatorname{DLDC}(3231)$, which can be optimized by maximizing the diversity gain using the rank criterion and maximizing the coding gain using the determinant criterion [17]. Consequently, we can generate
Table I

THE $P_{i n}=6$ COMPONENT CODES OF THE IR-PDLDC SCHEME OF FIGURE 1 GENERATED FOR A MIMO SYSTEM HAVING $M=3$ TRANSMIT AND $N=2$ RECEIVE ANTENNAS.

\begin{tabular}{|c|c|c|c|c|c|c|c|}
\hline Index & $M$ & $N$ & $T$ & $Q$ & Rate & $D$ & $j$ \\
\hline 0 & 3 & 2 & 3 & 1 & 0.33 & 6 & 0 \\
1 & $\vdots$ & $\vdots$ & $\vdots$ & 2 & 0.67 & 6 & 1 \\
2 & $\vdots$ & $\vdots$ & $\vdots$ & 3 & 1 & 6 & 1 \\
3 & $\vdots$ & $\vdots$ & $\vdots$ & 4 & 1.33 & 6 & 1 \\
4 & $\vdots$ & $\vdots$ & $\vdots$ & 5 & 1.67 & 6 & 1 \\
5 & $\vdots$ & $\vdots$ & $\vdots$ & 6 & 2 & 6 & 1 \\
\hline
\end{tabular}

different-rate DLDC components by gradually increasing $Q$ in order to increase the rate. Hence, by increasing the value of $Q$ and maximizing the diversity gain and coding gain for each resultant $\operatorname{DLDC}(M N T Q)$ component of the IR-PDLDC, we can generate a set of meritorious DLDCs. Since $Q$ transmitted symbols are jointly detected, low $Q$ values are desirable for the sake of maintaining a low complexity. The resultant $P_{i n}=6$ component codes designed for our IR-PDLDC scheme are listed in Table I.

\section{The number of inner iterations $(j)$}

The number of 'inner' iterations between the MMSE detector and the precoder required for each PDLDC component of Table I is also optimized for the sake of achieving the maximum throughput.

The so-called area property [16] of EXIT charts may be formulated by stating that the area under the 'outer' IRCC curve is approximately equal to its code rate $R_{\text {out }}$. Thus, if we assume that the area under the EXIT curve of an outer code can be perfectly matched to the area under the inner IR-PDLDC's EXIT curve at any $\operatorname{SNR} \rho$, then it is possible to approximate the maximum achievable rate of a serial concatenated scheme by evaluating the area under the EXIT curves, given the rate of the 'inner' PDLDC $R_{i n}$, which is expressed as:

$$
C(\rho)=\log _{2}(L) \cdot R_{\text {in }} \cdot R_{\text {out }},[\text { bits } / \text { sym } / H z]
$$

when $L$-PAM modulation is used.

Figure 2 quantifies the maximum achievable rates for three PDLDC components of Table I using different number of inner iterations $j$. For each set of comparisons, the maximum achievable rate of the DLDC quantified after the MMSE detector of Figure 1 is plotted as a benchmarker. For the rate $R_{5,3236}=2$ scheme, we observe a clear gap between the maximum achievable rate quantified both before and after the precoder, when employing no inner iterations i.e. for $j=0$. However, when we have $j=1$, the aforementioned rate loss is eliminated and a further increase of the number of inner iterations $j$ has only a modest additional rate improvement. For the rate $R_{2,3233}=1$ scheme of Table I, we observe in Figure 2 that although the aforementioned maximum achievable rate 


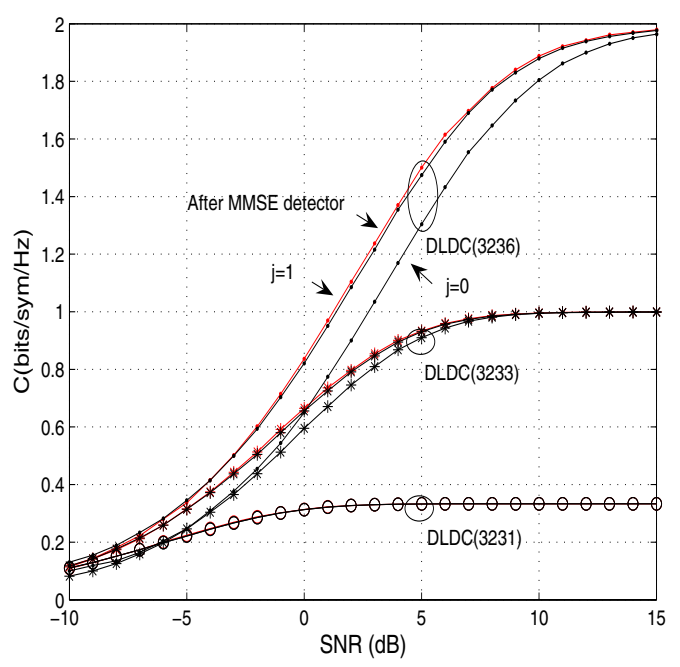

Figure 2. Comparison of the maximum achievable rates for the various PDLDC schemes of Table I having $j=0,1$ inner iterations, when using 2PAM modulation and an MMSE detector.

loss of employing the precoder is still present, when employing $j=0$ inner iterations, the associated discrepancy is narrower than that seen for the PDLDC $(3236)$ scheme. By contrast, for the $R_{0,3231}=0.33$ scheme, there is no maximum achievable rate loss even in the absence of inner iterations.

The above observations are related to the EXIT characteristics of the DLDC MMSE decoding block. When a single symbol is transmitted, the resultant EXIT curve is a horizontal line, which is a property of the Gray labelling employed [14]. Therefore, regardless of the number of inner iterations employed, the MMSE detector of Figure 1 always outputs the same extrinsic information. When $Q$ is increased, the resultant EXIT curve become more steep, therefore higher extrinsic information can be obtained upon increasing the a-priori information by using a higher number of inner iterations. Therefore, the resultant maximum achievable rate of PDLDCs observed in Figure 2 has an increasing discrepancy with respect to the DLDCs without precoders, when a higher value of $Q$ is employed. We observe that for the component PDLDCs of Table I, where we have $Q>1$, employing $j=1$ inner iteration will enable the system to attain $99 \%$ of the maximum rate quantified before the precoders.

\section{Optimizing the weighing coefficient vectors}

Apart from the specific shape of the component EXIT curves, the aggregate IR-PDLDC scheme's EXIT curve characterized in Equation (5) is also affected by the weighting coefficients $\lambda_{i}$, where $\lambda_{i}$ represents the specific fraction of information bits fed into the IR-PDLDC encoder/decoder of Figure 1. Therefore, $\lambda$ has to satisfy $\sum_{i=1}^{P_{i n}} \lambda_{i}=1$, where $\lambda_{i} \in[0,1]$. Similarly, the IRCC's weighting coefficient vector $\gamma$ quantifying the fraction of the LLRs $I_{A}\left(c_{1}\right)$ fed into the IRCC decoder has to obey $\sum_{i=1}^{P_{\text {out }}} \gamma_{i}=1$, where $\gamma_{i} \in[0,1]$. The resultant aggregate rates $R_{i n}$ and $R_{\text {out }}$ are given as follows:

$$
\left\{\begin{array}{cc}
1 / R_{\text {in }} & =\sum_{i=1}^{P_{\text {in }}} \frac{1}{R_{(i, D L D C)}} \cdot \lambda_{i} \\
R_{\text {out }} & =\sum_{i=1}^{P_{\text {out }}} R_{(i, I R C C)} \cdot \gamma_{i}
\end{array}\right.
$$

Our aim is to achieve the maximum throughput at a specific SNR $\rho$, while maintaining an infinitesimally low BER. Hence, we conducted an exhaustive search for all the possible combinations of $\gamma$ and $\boldsymbol{\lambda}$ under the following constraints:

- An open convergence tunnel exists in the EXIT chart;

- The open EXIT tunnel area is maximized, for the sake of minimizing the number iterations required;

- Throughput of Equation (8) is maximized;

- $\sum_{i=1}^{P_{\text {in }}} \lambda_{i}=1$ and $\sum_{i=1}^{P_{\text {out }}} \gamma_{i}=1$ have to be satisfied.

In our investigations, we found that a weighting factor search step-size of 0.05 applied across the range of the entire space was sufficient to explore the proposed system's irregularity.

More explicitly, having an open EXIT tunnel ensures that an infinitesimally low BER can be achieved with the aid of iterative detectors. Furthermore, having a larger EXIT tunnel area potentially requires less iterations to achieve the target BER. We maximized the throughput according to Equation (8), when tentatively using all the possible weighting coefficient combinations, given the step-size of 0.05 .

\section{SIMULATION RESULTS}

In this section, we present our numerical results characterizing the proposed scheme of Figure 1 designed for maximizing the achievable throughput, while maintaining an infinitesimally low BER. For all the simulations, 2PAM i.e. BPSK modulation is employed and the first interleaver of Figure 1 is set to have a length of $10^{6}$ bits. We employed a total of $P=12$ component codes, while the IR-PDLDC scheme used the $P_{i n}=6$ component codes of Table I. However, as a result of the optimization procedure, the IRCC contains only $P_{\text {out }}=6$ component codes, namely those having a rate of $R_{i, I R C C}=[0.1,0.25,0.4,0.55,0.7,0.9]$.

Figure 3 presents the EXIT chart and the corresponding decoding trajectory of our IRCC-coded IR-PDLDC scheme designed for $\rho=-2 \mathrm{~dB}$ using an MMSE detector, when communicating over Rayleigh fading channels having a normalized Doppler frequency of $f_{d}=10^{-2}$. The six dotted curves represent the EXIT curves of the six IR-PDLDC component codes of Table I and the dashed lines denote the EXIT curves recorded for the above-mentioned set of IRCC components. The shape of all the six dotted curves is similar, since they are all combined with the same precoder, while the are under the curves is proportional to their code rates. The solid lines represent the aggregate EXIT curves of the IRCC and IR-PDLDC schemes having the optimized weighting coefficients of $\lambda=[0$, $0,0,0,0.3,0.7$, $\gamma=\left[\begin{array}{llllll}0.4 & 0.25 & 0.15 & 0.05 & 0.1 & 0.05\end{array}\right]$, which were found using the method presented in Section III-D. The resultant maximum rate is $C(-2 d B)=0.5755$ [bits/sym/Hz]. By simultaneously maximizing $C(-2 d B)$ and the EXIT tunnel area, the optimized EXIT curves of Figure 3 exhibit a narrow open tunnel, where the decoding trajectory shows that $k=49$ outer iterations were required for reaching the point $(1.0,1.0)$ of perfect convergence. 


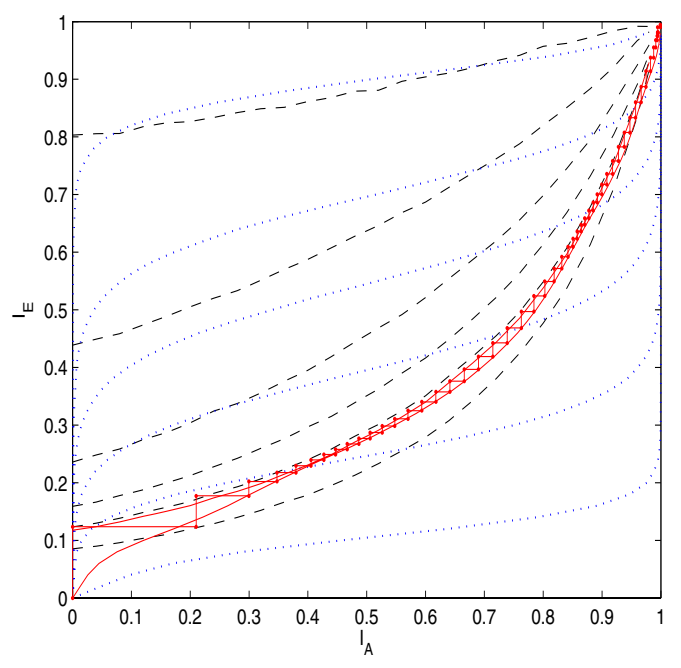

Figure 3. EXIT chart and the decoding trajectory of the IRCC-coded IRPDLDC scheme of Figure 1 recorded at $\rho=-2 \mathrm{~dB}$ using an MMSE detector, while having a normalized Doppler frequency of $f_{d}=10^{-2}$.

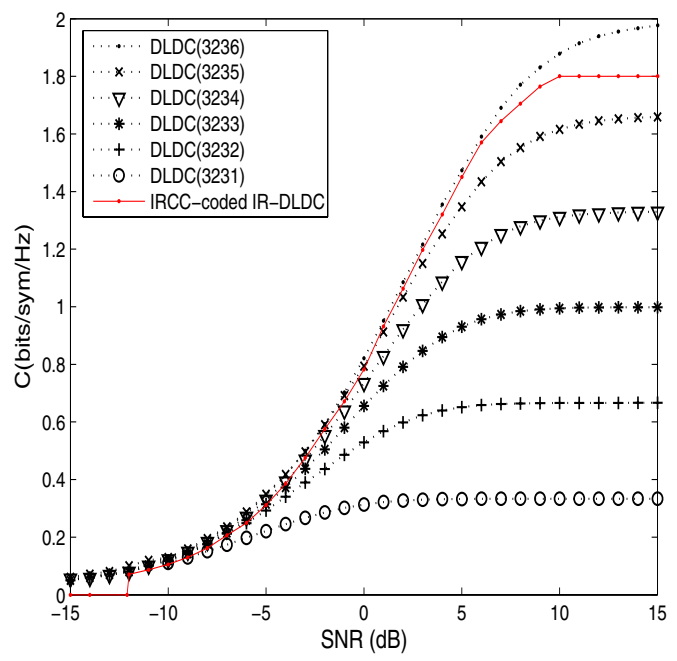

Figure 4. The maximum rates achieved by the IRCC-coded IR-PDLDC scheme of Figure 1 using an MMSE detector, while having a normalized Doppler frequency of $f_{d}=10^{-2}$.

Naturally, the same design process can be extended to other SNR values. Figure 4 plots the maximum rates achieved by the proposed irregular scheme of Figure 1 using an MMSE detector, while having a normalized Doppler frequency of $f_{d}=10^{-2}$. Each point on the solid line of Figure 4 was designed to achieve the maximum rate using a specific weighting coefficient vector pair $[\lambda, \gamma]$ at the corresponding SNR values, although they are not included here owing to the lack of space. The dotted lines in Figure 4 represent the maximum achievable rates of the DLDC component codes of Table I. The proposed scheme is capable of operating at SNRs in excess of $\rho=-12 \mathrm{~dB}$, while simultaneously approaching the maximum possible rate and maintaining an infinitesimally low BER. However, the attainable rate is limited to $1.8[\mathrm{bits} / \mathrm{sym} / \mathrm{Hz}]$ for
SNRs beyond $\rho=10 \mathrm{~dB}$. Clearly, a further rate increase can be achieved, when higher-rate DLDC components are employed.

\section{CONCLUSION}

In this paper, we proposed a novel IRCC-coded IR-PDLDC scheme and demonstrated that it is capable of reliably providing high rates for SNRs in excess of $\rho=-12 \mathrm{~dB}$, while maintaining an infinitesimally low BER. A high design flexibility is achieved by facilitating the irregular design of both the outer and inner codes, where the iterative detector of Figure 1 is employed. The other coding blocks of Figure 1 and the choice of all the iterative decoding parameters were also optimized from a throughput maximization perspective with the aid of EXIT charts.

\section{REFERENCES}

[1] G. J. Foschini and M. J. Gans. On limits of wireless communications in a fading environment when using multiple antennas. Wireless Personal Communications, 6(3):311-335, 1998.

[2] I. E. Telatar. Capacity of multi-antenna Gaussian channels. European Transactions on Telecommunications, 10:585-595, 1999.

[3] L. Z. Zheng and D.N.C. Tse. Diversity and multiplexing: a fundamental tradeoff in multiple-antenna channels. IEEE Transactions on Information Theory, 49(5):1073-1096, 2003.

[4] B. Hassibi and B. M. Hochwald. High-rate codes that are linear in space and time. IEEE Transactions on Information Theory, 48(7):1804-1824, July 2002.

[5] R. W. Jr. Heath and A. J. Paulraj. Linear dispersion codes for MIMO systems based on frame theory. IEEE Transactions on Signal Processing, 50(10):2429-2441, October 2002.

[6] B. M. Hochwald and W. Sweldens. Differential unitary space-time modulation. IEEE Transactions on Communications, 48(12):2041-2052, 2000.

[7] V. Tarokh and H. Jafarkhani. A differential detection scheme for transmit diversity. IEEE Journal on Selected Areas in Communications, 18(7):1169-1174, 2000.

[8] B. Hassibi and B. M. Hochwald. Cayley differential unitary space-time codes. IEEE Transactions on Information Theory, 48(6):1485-1503, 2002.

[9] S. Benedetto, D. Divsalar, G. Montorsi, and F. Pollara. Serial concatenation of interleaved codes: performance analysis, design, and iterative decoding. IEEE Transactions on Information Theory, 44(3):909-926, 1998.

[10] M. Tüchler and J. Hagenauer. Exit charts of irregular codes. 36th Conference on Information Sciences and Systems (CISS), (Princeton University), pages 748-753, March 20-22, 2002.

[11] M. Tüchler. Design of serially concatenated systems depending on the block length. IEEE Transactions on Communications, 52(2):209-218, February 2004.

[12] S. ten Brink, J. Speidel, and R. H. Yan. Iterative demapping and decoding for multilevel modulation. IEEE Global Telecommunications Conference, GLOBECOM, 1:579-584, November 1998, Sydney Australia.

[13] L. Hanzo, T. H. Liew, and B. L. Yeap. Turbo Coding, Turbo Equalisation, and Space-Time Coding: For Transmission over Fading Channels. John Wiley and IEEE press, 2002.

[14] O. Alamri, N. Wu, and L. Hanzo. A differential turbo detection aided sphere packing modulated space-time coding scheme. IEEE Vehicular Technology Conference, 5:2474-2478, May 2006, Melbourne Australia.

[15] S. ten Brink. Designing iterative decoding schemes with the extrinsic information transfer chart. AE ̈̈ International Journal of Electronics and Communicatioons, 54:389-398, 2000.

[16] A. Ashikhmin, G. Kramer, and S. ten Brink. Extrinsic information transfer functions: model and erasure channel properties. IEEE Transactions on Information Theory, 50(11):2657-2673, 2004.

[17] V. Tarokh, N. Seshadri, and A. R. Calderbank. Space-time codes for high data rate wireless communication: performance criterion and code construction. IEEE Transactions on Information Theory, 44(2):744-765, 1998. 